Supporting Information for

\title{
Surface Engineering of 3D Topological Network for Ultrasensitive Piezoresistive Pressure Sensors
}

Hong Pan, ${ }^{\dagger}$ Guangzhong Xie, ${ }^{\dagger}$ Wenqian Pang, ${ }^{\dagger}$ Si Wang, ${ }^{\dagger}$ Yang Wang, ${ }^{\dagger}$ * Zhi Jiang, ${ }^{\ddagger}$ Xiaosong Du, Huiling Tai ${ }^{\dagger}$

${ }^{\dagger}$ State Key Laboratory of Electronic Thin Films and Integrated Devices, School of Optoelectronic

Science and Engineering, University of Electronic Science and Technology of China (UESTC),

Chengdu 610054, PR China

Electrical and Electronic Engineering and Information Systems, The University of Tokyo, 113-8656

Tokyo, Japan

*E-mail: Yang Wang (landlord@uestc.edu.cn) 


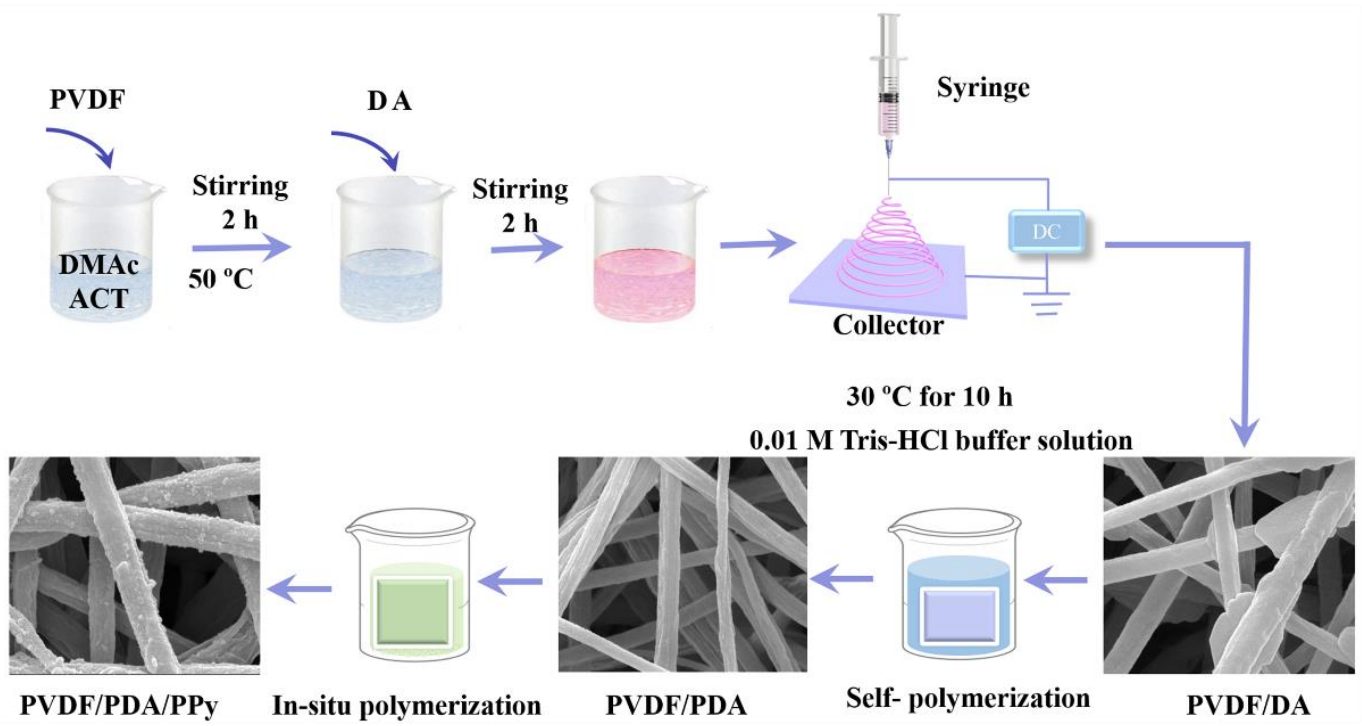

Scheme S1. The process for preparing the 3D PPP network film 


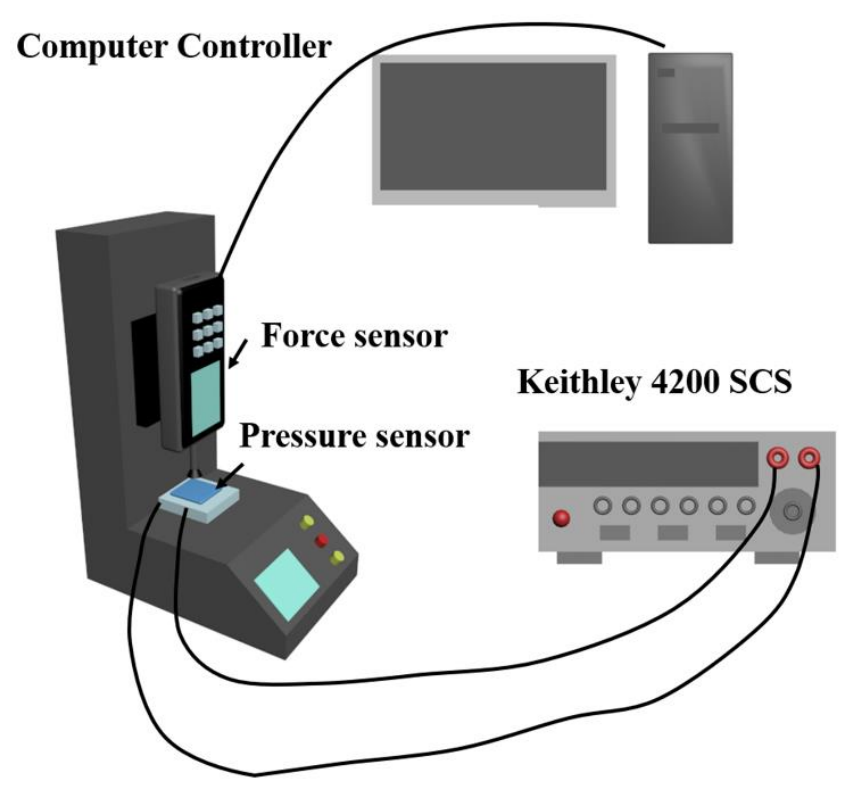

Figure S1. The measurement platform for static pressure 


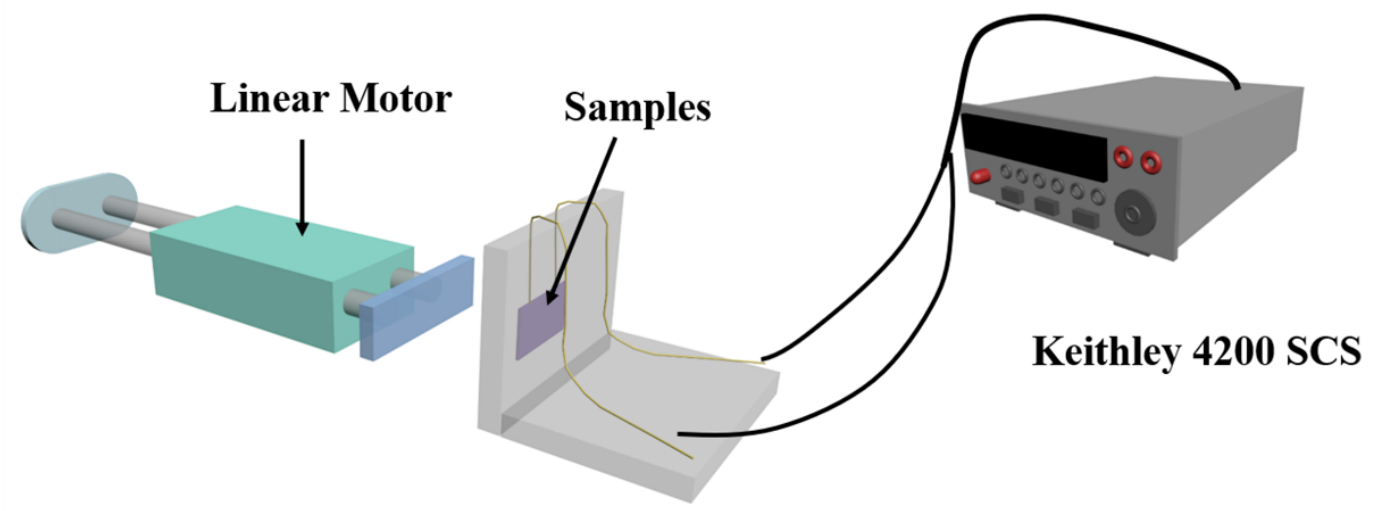

Figure S2. The measurement platform for dynamic pressure 


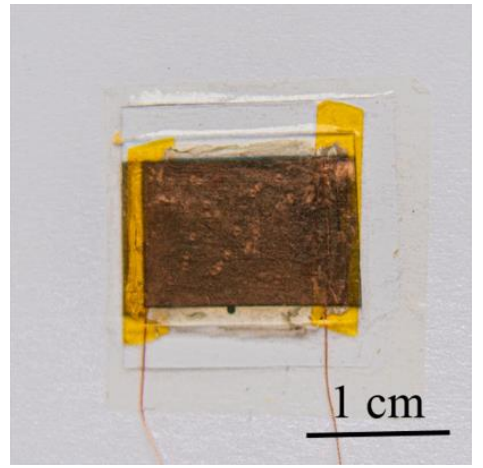

Figure S3. Photograph of the PPP-based pressure sensor. 


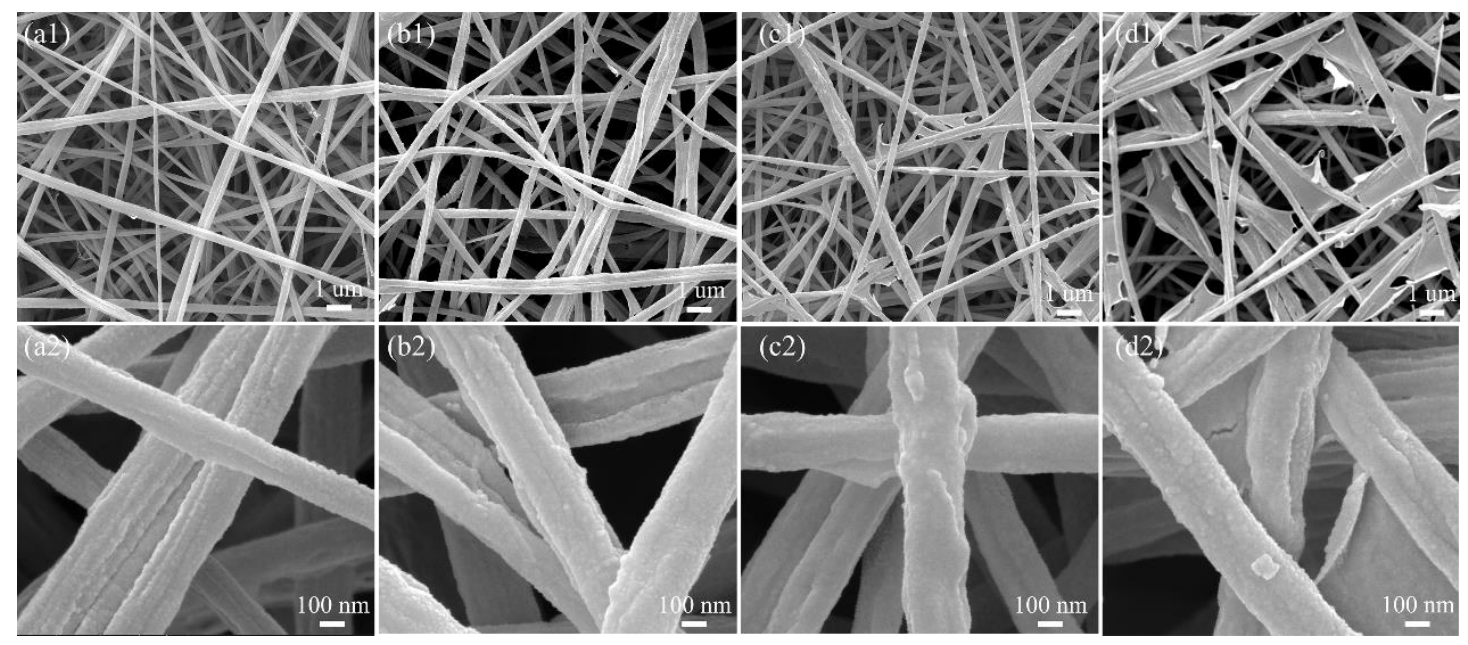

Figure S4. SEM images of the PVDF/DA nanofiber network immersing into the buffer solution for different treating time. (a1, a2): 6 h; (b1, b2): 10 h; (c1, c2): 24 h; (d1, d2): 30 h. 

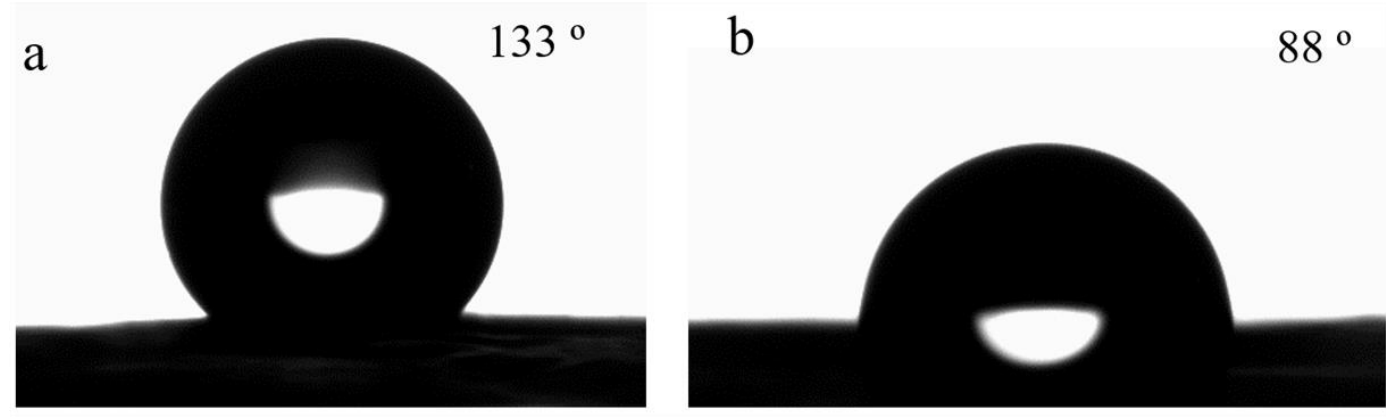

Figure S5. Static contact angles of DI water on (a) pure PVDF and (b) PVDF/PDA films, respectively. 


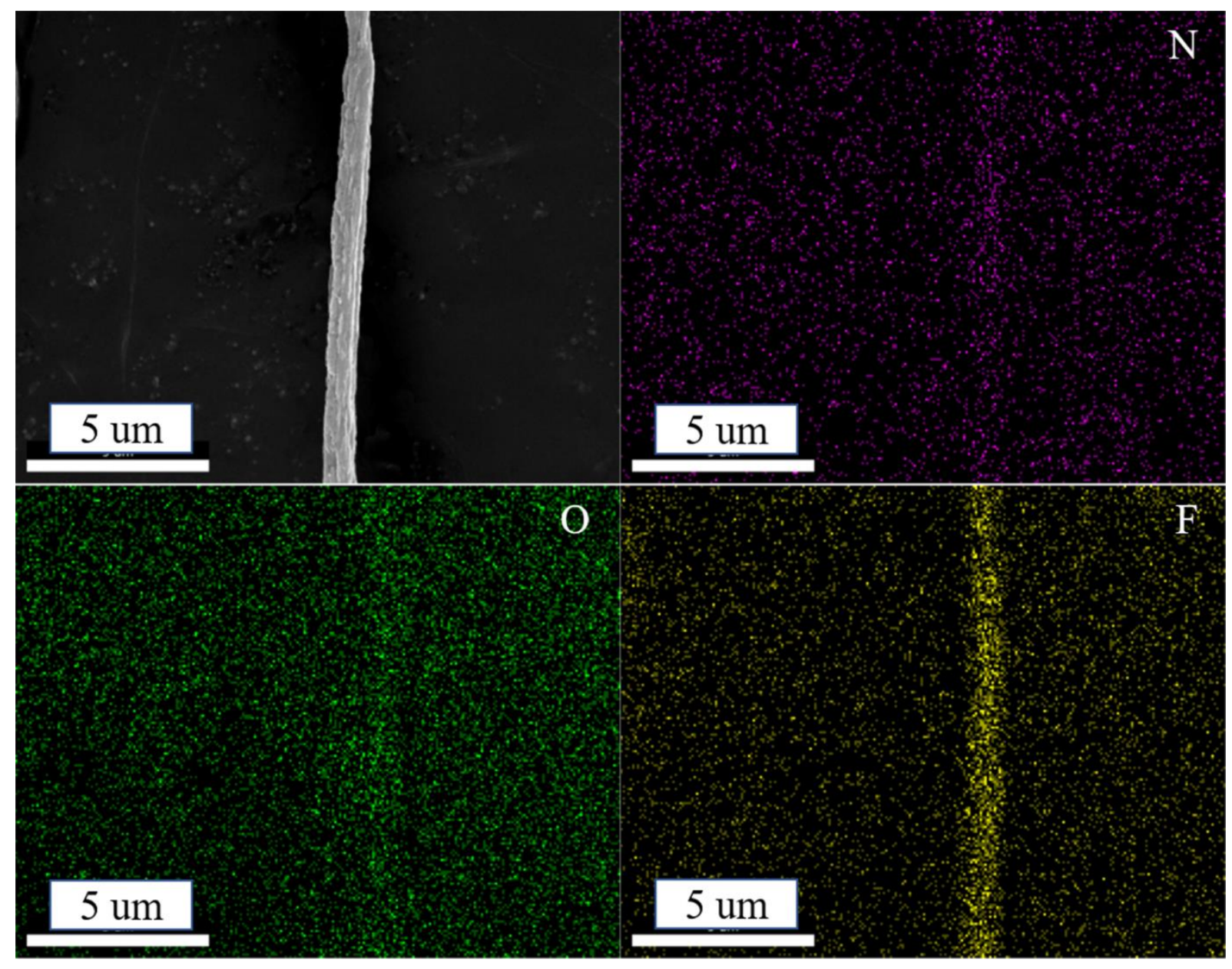

Figure S6. The EDS mapping images of N, O and F elements in one single PPP nanofiber 


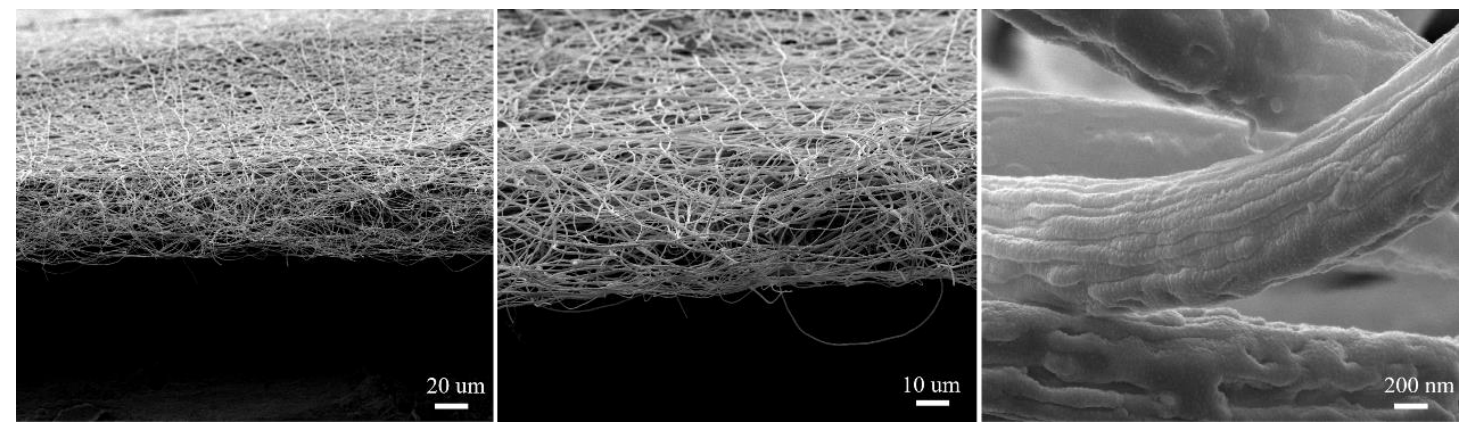

Figure S7. Cross-sectional SEM images of the PPP film. 


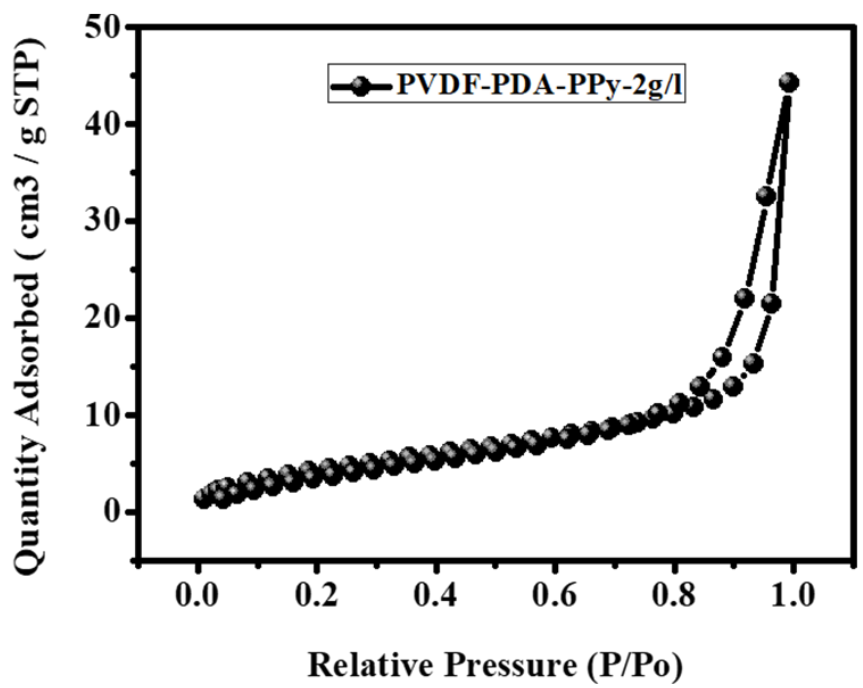

Figure S8. Quantity adsorbed curve of PPP film (with the Py concentration of $2 \mathrm{~g} / \mathrm{L}$ ). 

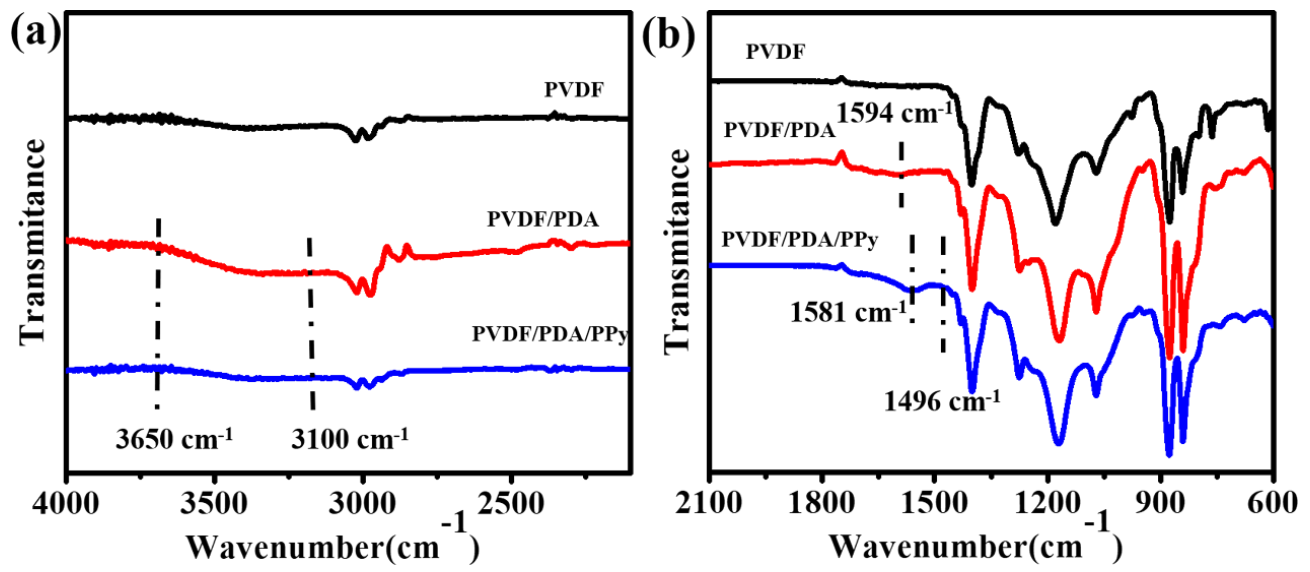

Figure S9. FTIR spectra of the different samples as indicated in the figures. (a) and (b) are the spectra in the high and low wavenumber ranges, respectively. 


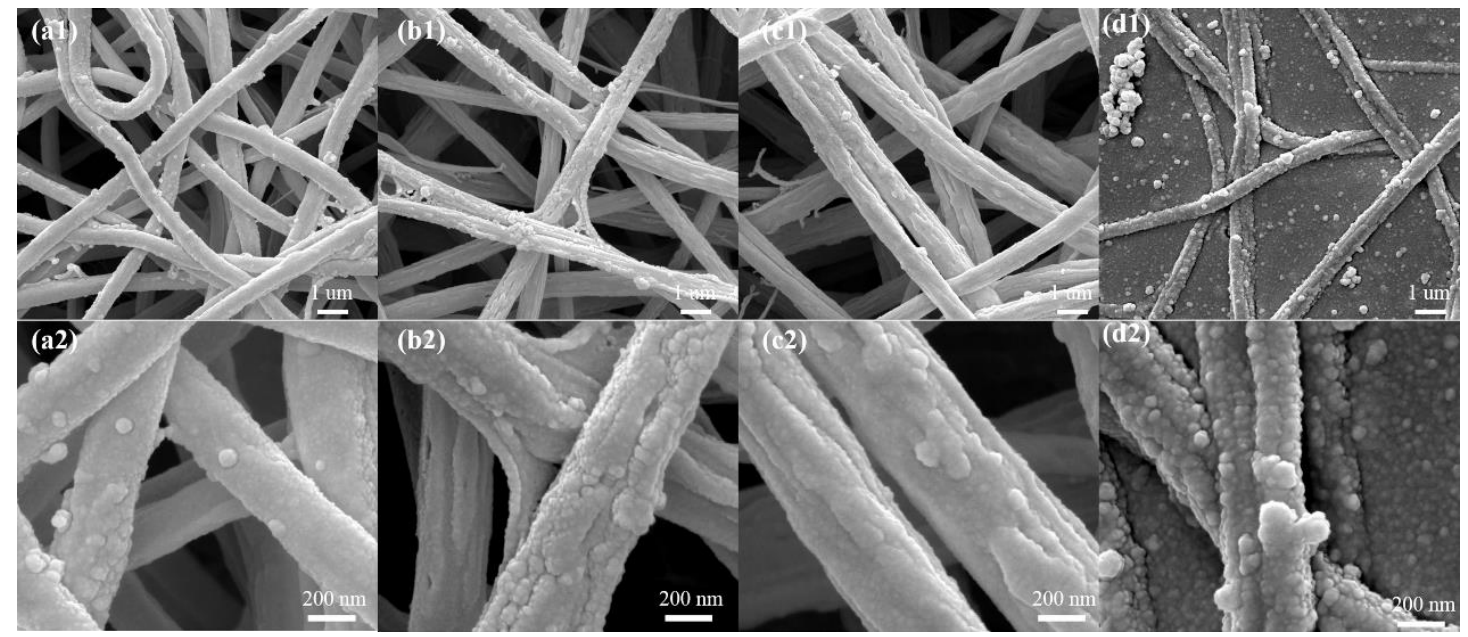

Figure S10. SEM images of PPP-1 (a1, a2), PPP-2 (b1, b2), PPP-3 (c1, c2), and PPP-4 (d1, d2), respectively. 


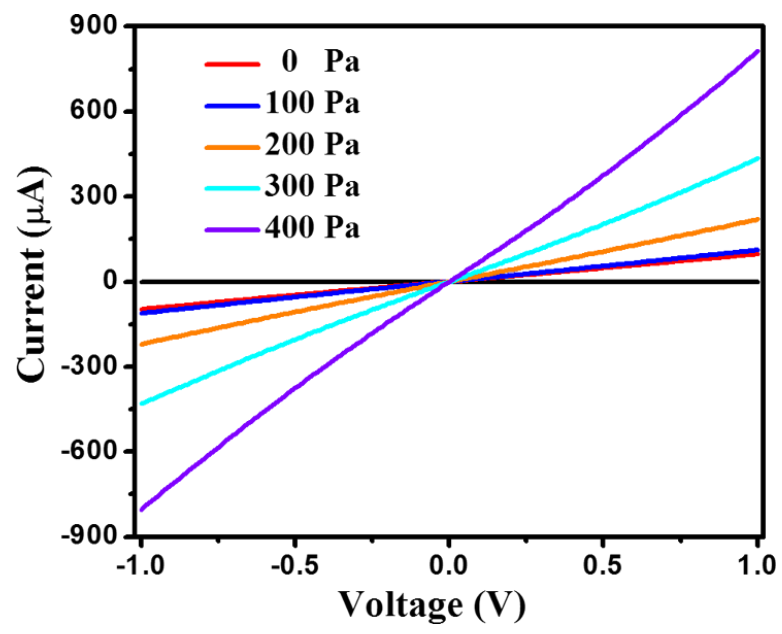

Figure S11. I-V curves of the PPP-3 based sensor under different pressures from $0 \mathrm{~Pa}$ to $400 \mathrm{~Pa}$ 

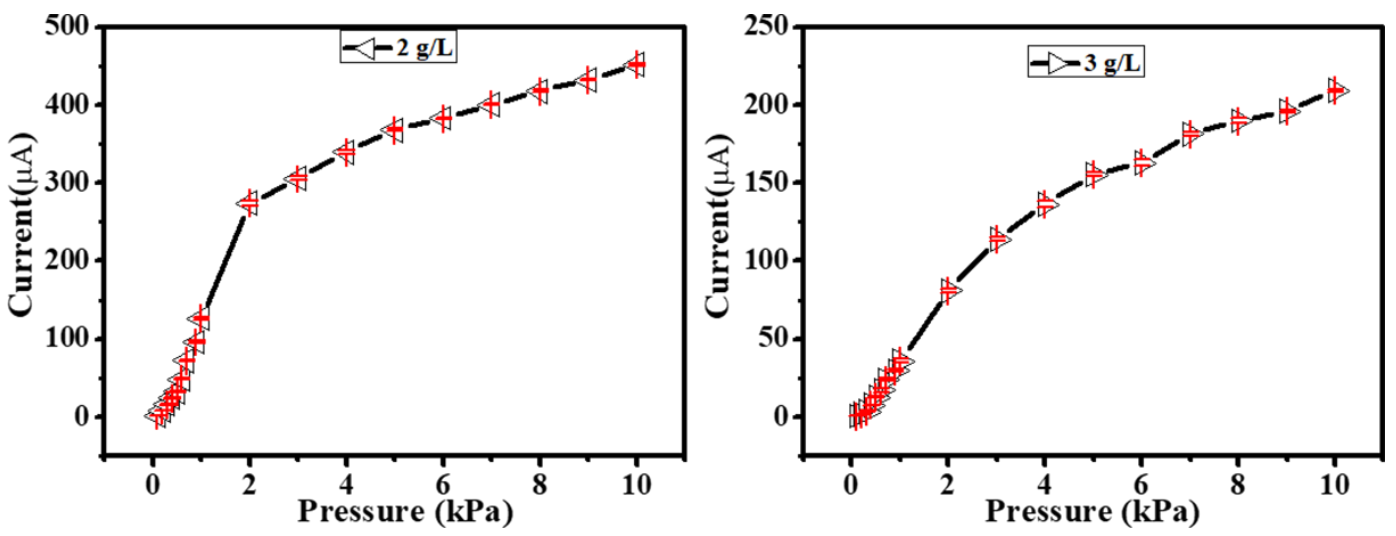

Figure S12. The sensing performance with the standard deviation for Figure $1 b$. 

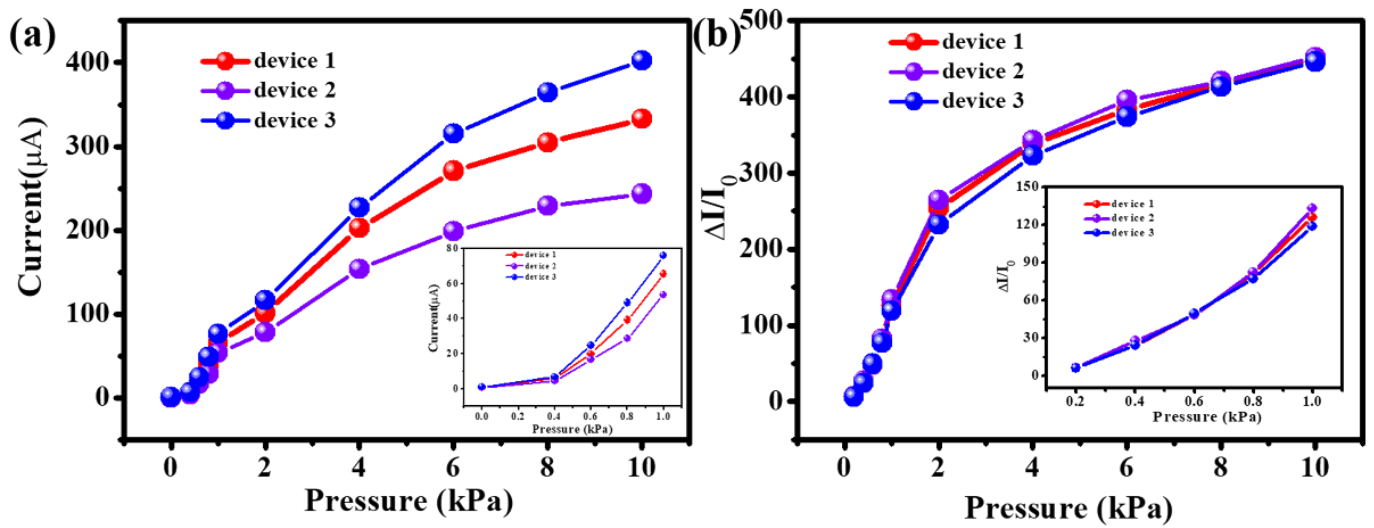

Figure S13. (a)Current vs. pressure curves and (b) the relative change of the current vs. pressure curves of three different devices. 

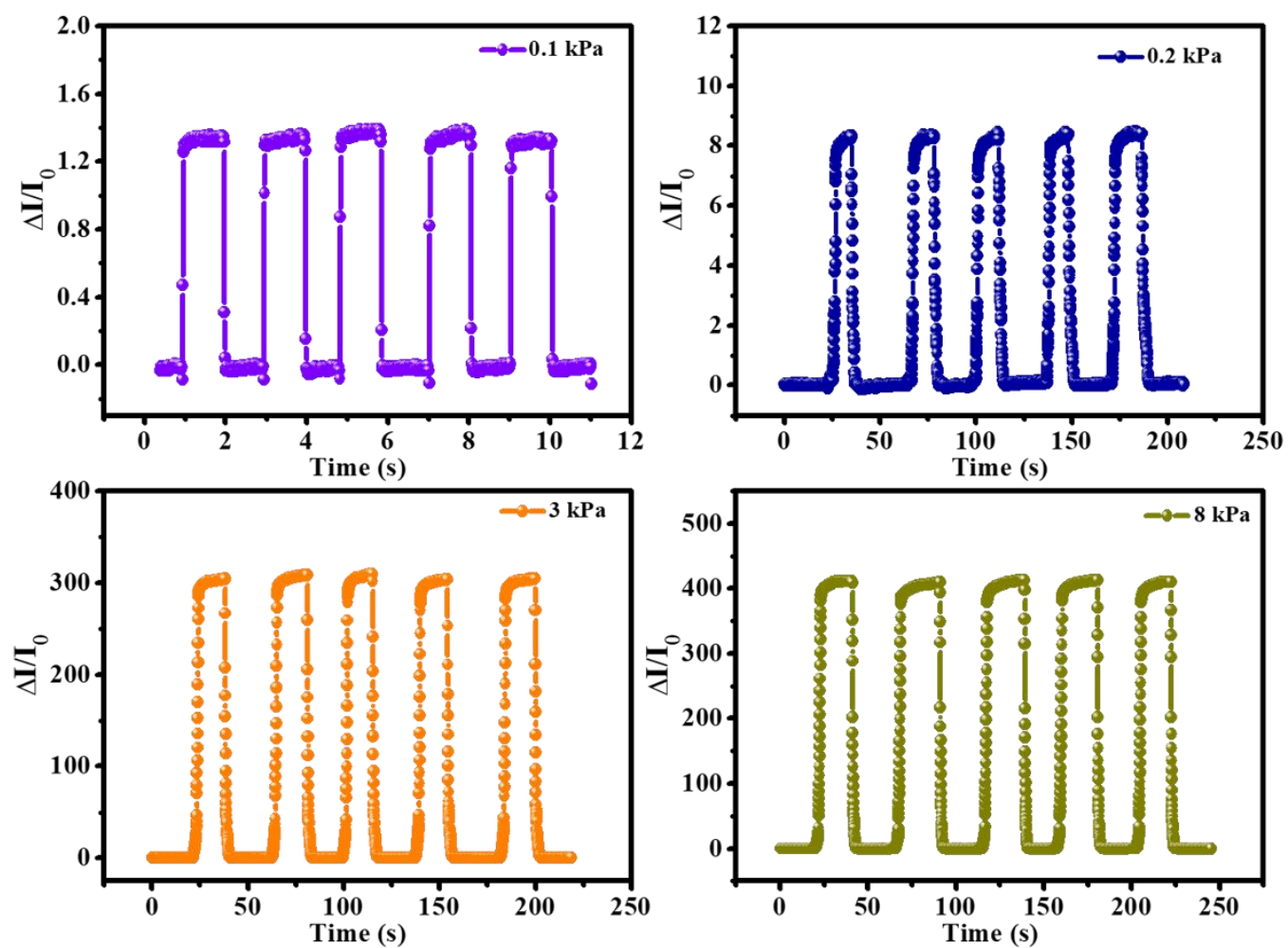

Figure S14. The relative current changes under different external pressure of $100 \mathrm{~Pa}, 200 \mathrm{~Pa}, 3 \mathrm{kPa}$, $8 \mathrm{kPa}$ for 5 cycles 


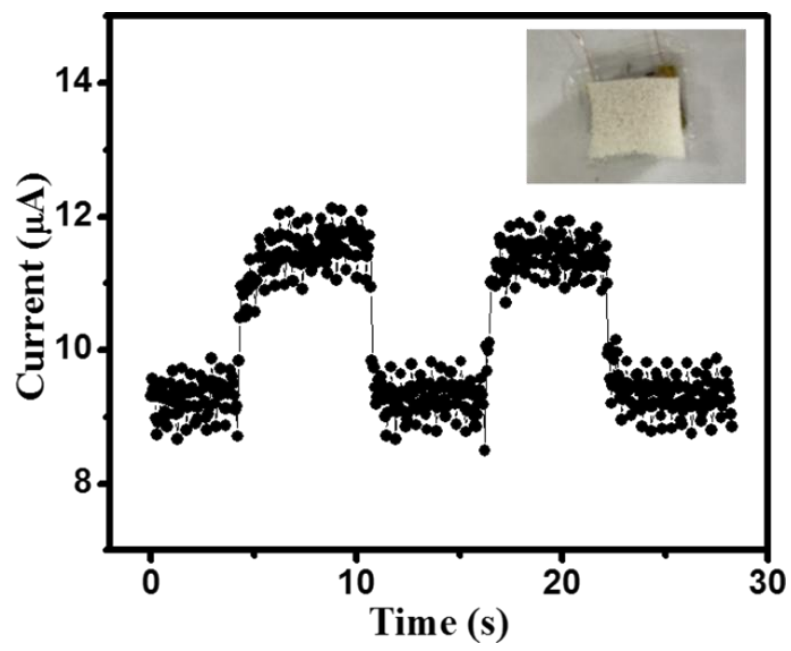

Figure S15. Two cycles of loading and unloading a block of sponge with a mass of about $25 \mathrm{mg}$, corresponding to $\sim 2.5 \mathrm{~Pa}$. 


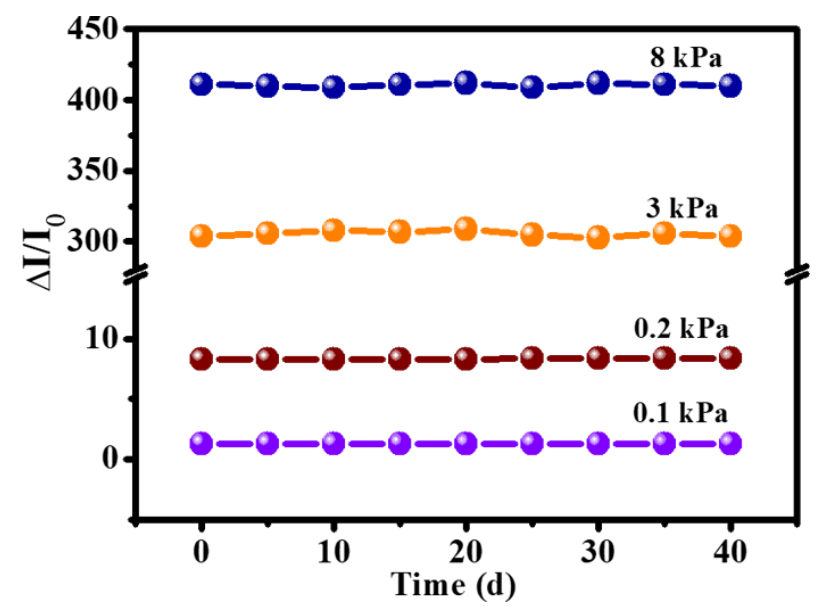

Figure S16. The temporal stability of device under various external pressures. 


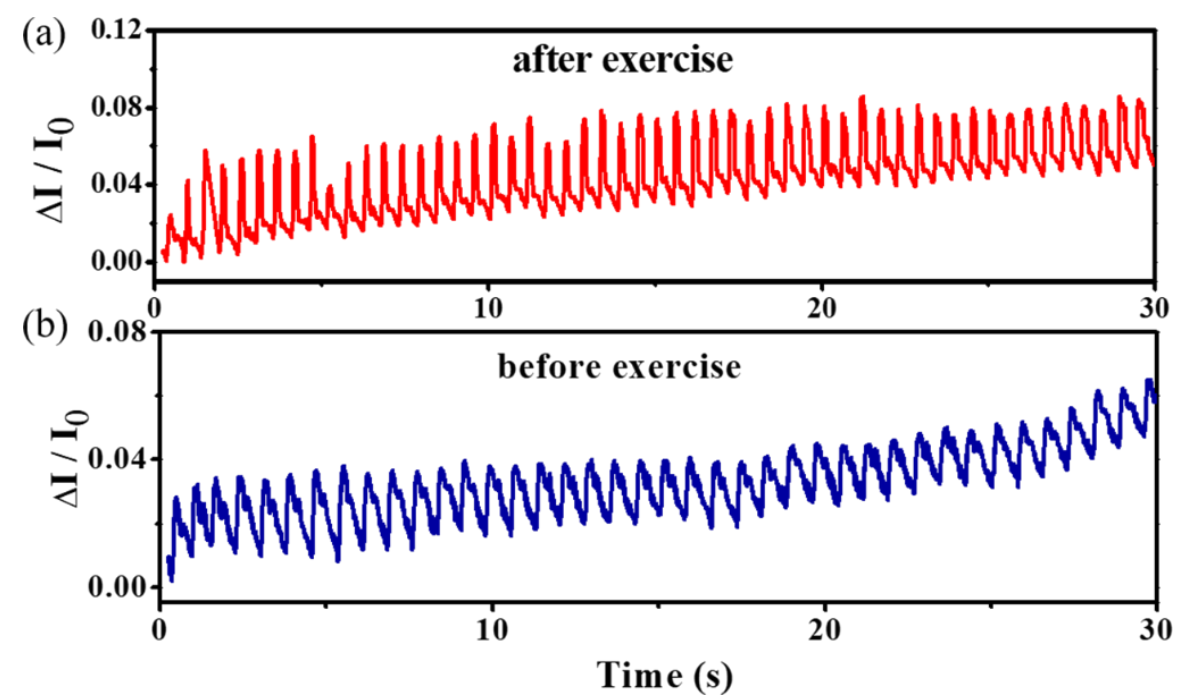

Figure S17. Real-time arterial pulse waves of a male (a) before and (b) after walking for $30 \mathrm{~s}$. 

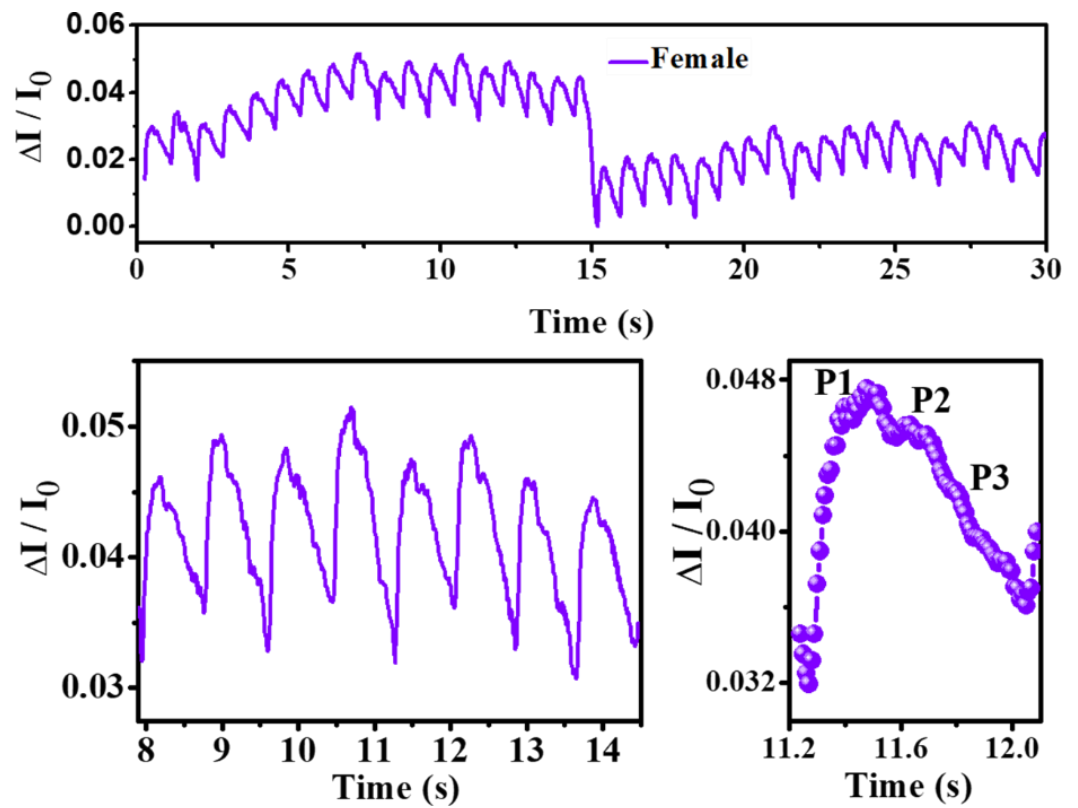

Figure S18. Real-time arterial pulse waves of a female. (age: 28; height: $158 \mathrm{~cm}$; weight: $48 \mathrm{~kg}$ ) 
Table S1. A comparison of sensitive performance among recently reported representative piezoresistive pressure sensors based on various 3D structure.

\begin{tabular}{|c|c|c|c|c|}
\hline Material & 3D structure & Sensitivity $\left[\mathrm{kPa}^{-1}\right]$ & Detection limit & Ref \\
\hline PVDF/RGO & nanofiber & 15.6 & $1.2 \mathrm{~Pa}$ & 1 \\
\hline PEDOT:PSS/PVDF & nanofiber & 13.5 & $1 \mathrm{~Pa}$ & 2 \\
\hline PPy/PVA & nanofiber & 1.24 & $1.3 \mathrm{~Pa}$ & 3 \\
\hline Au nanowires & paper & $>1.14$ & $13 \mathrm{~Pa}$ & 4 \\
\hline Mxene nanosheet & paper & $0.55,3.81,2.52$ & $10.2 \mathrm{~Pa}$ & 5 \\
\hline PANI/C-RGO & PU foam & $\begin{array}{c}0.0021,-0.0044 \\
-0.0109\end{array}$ & $25 \mathrm{mg}$ & 6 \\
\hline RGO & PU sponge & 0.26 & $9 \mathrm{~Pa}$ & 7 \\
\hline MWNT-RGO ink & PU foam & 0.022 & $3.7 \mathrm{~Pa}$ & 8 \\
\hline CNTs & PDMS foam & 0.3 & $6 \mathrm{~Pa}$ & 9 \\
\hline CNTs & cotton & 14.4 & $2 \mathrm{~Pa}$ & 10 \\
\hline Ag nanoparticle & Keviar fiber & 0.21 & $8 \mathrm{mg}$ & 11 \\
\hline RGO/Mxene & aerogel & 22.56 & $10 \mathrm{~Pa}$ & 12 \\
\hline PVDF/PDA/PPy & nanofiber & 139.9 & $0.9 \mathrm{~Pa}$ & This work \\
\hline
\end{tabular}




\section{REFERENCES}

(1). Lou, Z.; Chen, S.; Wang, L.; Jiang, K., Shen, G. An ultra-sensitive and rapid response speed graphene pressure sensors for electronic skin and health monitoring. Nano Energy 2016, 23, 237-244. (2). Kweon, O.Y.; Lee, S.J., Oh, J.H. Wearable high-performance pressure sensors based on threedimensional electrospun conductive nanofibers. NPG Asia Mater. 2018, 10(6), 540-551.

(3). Zhong, W.; Liu, Q.; Wu, Y.; Wang, Y.; Qing, X.; Li, M.; Liu, K.; Wang, W., Wang, D. A nanofiber based artificial electronic skin with high pressure sensitivity and 3D conformability. Nanoscale 2016, 8(24),12105-12112.

(4). Gong, S.; Schwalb, W.; Wang, Y.; Chen, Y.; Tang, Y.; Si, J.; Shirinzadeh, B., Cheng, W. A wearable and highly sensitive pressure sensor with ultrathin gold nanowires. Nat. Commun. 2014, 5(1), 53132-53139.

(5). Guo, Y.; Zhong, M.; Fang, Z.; Wan, P., Yu, G. A Wearable Transient Pressure Sensor Made with MXene Nanosheets for Sensitive Broad-Range Human-Machine Interfacing. Nano Lett. 2019, 19(2),1143-1150.

(6). Guo, Y., Guo, Z., Zhong, M., Wan, P., Zhang, W., \& Zhang, L. A Flexible Wearable Pressure Sensor with Bioinspired Microcrack and Interlocking for Full-Range Human-Machine Interfacing. Small, 2018, 14(44), 1803018.

(7). Yao, H.B.; Ge, J.; Wang, C.F.; Wang, X.; Hu, W.; Zheng, Z.J.; Ni, Y., Yu, S.H. A flexible and highly pressure-sensitive graphene-polyurethane sponge based on fractured microstructure design. Adv. Mater. 2013, 25(46), 6692-6698. 
(8). Tewari, A., Gandla, S., Bohm, S., McNeill, C. R., \& Gupta, D. Highly exfoliated MWNT-rGO ink-wrapped polyurethane foam for piezoresistive pressure sensor applications. ACS Appl. Mater. Interfaces, 2018, 10(6), 5185-5195

(9). Iglio, R., Mariani, S., Robbiano, V., Strambini, L., \& Barillaro, G. Flexible polydimethylsiloxane foams decorated with multiwalled carbon nanotubes enable unprecedented detection of ultralow strain and pressure coupled with a large working range. ACS appl. Mater. Interfaces, 2018, 10(16), 13877-13885.

(10). Liu, M.; Pu, X.; Jiang, C.; Liu, T.; Huang, X.; Chen, L.; Du, C.; Sun, J.; Hu, W., Wang, Z.L. Large-Area All-Textile Pressure Sensors for Monitoring Human Motion and Physiological Signals. Adv. Mater. 2017, 29(41),1703700-1703708.

(11). Lee, J.; Kwon, H.; Seo, J.; Shin, S.; Koo, J.H.; Pang, C.; Son, S.; Kim, J.H.; Jang, Y.H.; Kim, D.E., Lee, T. Conductive fiber-based ultrasensitive textile pressure sensor for wearable electronics. Adv. Mater. 2015, 27(15), 2433-2439.

(12). Ma, Y.; Yue, Y.; Zhang, H.; Cheng, F.; Zhao, W.; Rao, J.; Luo, S.; Wang, J.; Jiang, X.; Liu, Z.; Liu, N., Gao, Y. 3D Synergistical MXene/Reduced Graphene Oxide Aerogel for a Piezoresistive Sensor. ACS Nano 2018, 12(4), 3209-3216. 